Meta

Journal des traducteurs

Translators' Journal

\title{
Terminologies et nouvelles technologies
}

\section{Nathalie Gormezano et Sandrine Peraldi}

Volume 57, numéro 1, mars 2012

La CIUTI, chef de file pour la promotion de l'employabilité et de la recherche

CIUTI: Leader in Advocating Employability and Research

URI : https://id.erudit.org/iderudit/1012752ar

DOI : https://doi.org/10.7202/1012752ar

Aller au sommaire du numéro

\section{Éditeur(s)}

Les Presses de l’Université de Montréal

ISSN

0026-0452 (imprimé)

1492-1421 (numérique)

Découvrir la revue

Citer cet article

Gormezano, N. \& Peraldi, S. (2012). Terminologies et nouvelles technologies. Meta, 57(1), 248-263. https://doi.org/10.7202/1012752ar

\section{Résumé de l'article}

À l'ère de la mondialisation, les modes d'accès à l'information sont en pleine mutation, que ce soit dans l'apprentissage ou dans l'exploitation des connaissances. La gestion de l'information est d'autant plus complexe qu'elle se conjugue avec la gestion d'un temps qui semble " accéléré ", en raison, d'une part, des relations multiples entre les systèmes et les individus et, d'autre part, de la demande de plus en plus pressante de rapidité dans la gestion des affaires, ceci afin de limiter les coûts. Dans cet univers mondialisé, mouvant, multiculturel et multilingue, la recherche en traductologie apparaît comme un lieu où peuvent être élaborées des propositions de solutions au service des acteurs de ce monde en mouvement. De fait, la gestion des connaissances dans des univers culturels différents est une activité de recherche de longue date dans le domaine de la traductologie ; la terminologie moderne donne lieu, depuis plusieurs années, à des développements intéressants autour des bases de données ; la linguistique, notamment grâce à la sémantique, a initié depuis quelques années également des travaux sur les bases de connaissances et les cartes sémantiques intégrées. De leur côté, les sciences des nouvelles technologies et en particulier la recherche sur les systèmes d'information ont développé des systèmes de bases de connaissances multimédias orientées métiers. C'est au regard de ces travaux et de leurs possibles interactions que le centre de recherche de l'ISIT, le CRATIL (Centre de recherche appliquée sur la traduction, l'interprétation et le langage) a mis en place des projets de recherche pluridisciplinaires où les nouvelles technologies et les terminologies liées à des métiers spécifiques sont devenues les outils de l'innovation au service de la société de la connaissance multilingue et multiculturelle.
Ce document est protégé par la loi sur le droit d'auteur. L’utilisation des services d’Érudit (y compris la reproduction) est assujettie à sa politique d'utilisation que vous pouvez consulter en ligne.

https://apropos.erudit.org/fr/usagers/politique-dutilisation/ 


\title{
LA TERMINOLOGIE DANS LES PROGRAMMES DE TRADUCTION
}

\section{Terminologies et nouvelles technologies}

\author{
NATHALIE GORMEZANO \\ ISIT, Paris, France \\ n.gormezano@isit-paris.fr
}

SANDRINE PERALDI

ISIT, Paris, France

s.peraldi@isit-paris.fr

\section{RÉSUMÉ}

À l'ère de la mondialisation, les modes d'accès à l'information sont en pleine mutation, que ce soit dans l'apprentissage ou dans l'exploitation des connaissances. La gestion de l'information est d'autant plus complexe qu'elle se conjugue avec la gestion d'un temps qui semble «accéléré», en raison, d'une part, des relations multiples entre les systèmes et les individus et, d'autre part, de la demande de plus en plus pressante de rapidité dans la gestion des affaires, ceci afin de limiter les coûts. Dans cet univers mondialisé, mouvant, multiculturel et multilingue, la recherche en traductologie apparaît comme un lieu où peuvent être élaborées des propositions de solutions au service des acteurs de ce monde en mouvement. De fait, la gestion des connaissances dans des univers culturels différents est une activité de recherche de longue date dans le domaine de la traductologie; la terminologie moderne donne lieu, depuis plusieurs années, à des développements intéressants autour des bases de données; la linguistique, notamment grâce à la sémantique, a initié depuis quelques années également des travaux sur les bases de connaissances et les cartes sémantiques intégrées. De leur côté, les sciences des nouvelles technologies et en particulier la recherche sur les systèmes d'information ont développé des systèmes de bases de connaissances multimédias orientées métiers. C'est au regard de ces travaux et de leurs possibles interactions que le centre de recherche de I'ISIT, le CRATIL (Centre de recherche appliquée sur la traduction, l'interprétation et le langage) a mis en place des projets de recherche pluridisciplinaires où les nouvelles technologies et les terminologies liées à des métiers spécifiques sont devenues les outils de l'innovation au service de la société de la connaissance multilingue et multiculturelle.

\footnotetext{
ABSTRACT

In the age of globalization, means of access to information are undergoing a radical transformation be it in a learning environment or in the use of knowledge. Information management is all the more complex since it goes hand in hand with time-management which seems to be accelerated due to, on the one hand, multi-relations between systems and individuals and on the other hand, the growing urgency for speed in treating cost issues in business management. In this globalized, shifting, multicultural and multilingual universe, research in the field of translation studies appears to be an area in which propositions of solutions to aid those involved in this ever-changing universe can be elaborated. Knowledge management in different cultural universes is a long-standing research activity in the realm of translation studies. For the past few years, classic terminology has resulted in interesting developments regarding databases. On the same note, linguistics and particularly semantics have been key domains of research for knowledge
} 
databases and integrated semantic maps. As for the sciences of new technologies and, in particular, research carried out on information systems, career-oriented multimedia knowledge databases have been developed. It is in this light that this research and its possible interaction with ISIT's research centre, CRATIL, put in place multidisciplinary research projects in which terminology related to specific careers and new technology have become tools of innovation in helping a multilingual and multicultural society.

\section{MOTS-CLÉS/KEYWORDS}

terminologies, interculturel, nouvelles technologies, sémantique, bases de connaissances terminology, intercultural, new technologies, semantics, knowledge databases

Dans un univers mondialisé, mouvant, multiculturel et multilingue, la recherche en traductologie apparaît comme un lieu où peuvent être élaborées des propositions de solutions au service des acteurs d'un monde en mouvement. La gestion des connaissances dans des univers culturels différents est une activité de recherche de longue date dans le domaine de la traductologie. Par ailleurs, la terminologie moderne donne lieu, depuis plusieurs années, à des développements intéressants autour des bases de données (Meyer, Skuce, et al. 1992; Szulman, Biébow, et al. 2001 ; Pecman, Juilliard, et al. 2010). Pour sa part, la linguistique, notamment grâce à la sémantique, a initié depuis quelques années également des travaux sur les bases de connaissances et les cartes sémantiques intégrées (Aussenac-Gilles et Condamines 2001). Enfin, les sciences des nouvelles technologies, et en particulier la recherche sur les systèmes d'information, ont développé des systèmes de bases de connaissances multimédias créées et mise au service des différents métiers (Aussenac-Gilles 1999).

C'est au regard de ces travaux et de leurs possibles interactions que le centre de recherche de l'ISIT, le CRATIL (Centre de recherche appliquée sur la traduction, l'interprétation et le langage) a mis en place des projets de recherche pluridisciplinaires où les terminologies liées à des métiers spécifiques et les nouvelles technologies sont devenues les outils de l'innovation, au service de la société de la connaissance multilingue et multiculturelle.

\section{De la terminologie aux terminologies}

\subsection{La terminologie face aux métamorphoses du monde}

La terminologie moderne telle qu'elle est apparue au milieu du $\mathrm{xx}^{\mathrm{e}}$ siècle a été créée à des fins de communication scientifique et technique. Elle avait pour rôle d'accompagner les progrès scientifiques, technologiques et industriels afin de faciliter les échanges internationaux et de lever les ambiguités et les confusions autour de la langue technique. En effet, Eugen Wüster, considéré comme le fondateur de la Théorie Générale de la Terminologie (TGT), était un fervent partisan d'une communication professionnelle rationalisée et sans ambiguïté: «Le travail terminologique commun ne doit pas se limiter à constater ce qui est. Il doit viser à unifier et à améliorer l'usage qui est si contradictoire ${ }^{1} »$ (Wüster 1979/1985: 89, cité et traduit par Candel 2004: 20). Malgré les nombreuses critiques à l'encontre de la théorie classique, parmi lesquelles la dimension extrêmement prescriptive de la TGT, l'idéal de biunivocité et monosémie et la démarche onomasiologique (Gambier 1991; Gaudin 1993, 2003; Temmerman 2000), cette recherche d'uniformisation et d'internationalisation consti- 
tue toujours une des missions dévolues de la terminologie. Nous en voulons pour preuve l'existence, de par le monde, de commissions de terminologie, d'associations, d'organismes de normalisation qui gèrent le sens et l'usage des unités terminologiques. Le travail de ces commissions a pour objectif de permettre à la langue des pays concernés de suivre les évolutions sociales, politiques, mais aussi technoscientifiques et de s'adapter au réel.

Ainsi, à l'appui de cette assertion, les institutions européennes sont dotées d'un service de terminologie portant sur les 27 langues de l'Union et travaillent en parallèle à l'élaboration et à la mise à jour de base de données, à l'instar de IATE (Interactive Terminology for Europe) ${ }^{2}$ et EUROVOC ${ }^{3}$. Les Nations Unies arborent également une base de référence UNTERM (United Nations Multilingual Terminology Database $)^{4}$. Pour l'univers politique et institutionnel, l'activité terminologique fait en effet partie intégrante des actions de communication et de diffusion des informations. Il est à noter d'ailleurs que celle-ci est très souvent englobée dans le processus plus vaste de la normalisation et de l'aménagement linguistiques ${ }^{5}$. À titre d'exemple, l'ensemble des mesures visant à protéger la langue française de l'invasion des anglicismes relève de ce processus d'aménagement. Les politiques linguistiques œuvrent également à la protection et à une meilleure intégration des populations nationales, ethniques et minoritaires, que ce soit à l'échelle d'un pays, de l'Europe ou du monde. C’est pourquoi, malgré la connotation souvent péjorative attribuée aux activités normalisatrices ${ }^{6}$, nombre d'organismes internationaux reconnaissent le potentiel positif de l'aménagement terminologique systématique en la matière. Ce dernier permettrait, en effet, d'appuyer des politiques d'information, du savoir, d'innovation ainsi que des stratégies d'éducation:

Les personnes dont la langue maternelle n'est pas (ou n'est pas suffisamment) développée du point de vue de la terminologie et des langues de spécialité (LS), ou celles à qui on refuse le droit à l'éducation, à la formation, à l'accès à l'information ou à l'interaction en milieu de travail dans leur langue maternelle, ont tendance à être défavorisées. [...] Dans la plupart des cas, une langue dont l'utilisation se limite au folklore ou au milieu familial ne pourra convenir à la communication professionnelle. Dans une même veine, les locuteurs d'une langue dont la terminologie n'est pas à jour dans une spécialité donnée risquent de perdre progressivement la capacité de communiquer à ce sujet dans leur langue. (UNESCO 2005: $\mathrm{V}^{7}$ )

Une approche qui va de pair avec l'idée que l'accélération de la mondialisation observée aujourd'hui doit systématiquement s'accompagner de mesures de localisation ; c'est-à-dire que la traduction et l'adaptation doivent respecter les normes culturelles et linguistiques locales. Nous y reviendrons.

Outre les domaines politiques et économiques, la terminologie occupe également la scène scientifique et industrielle. En effet, le développement des sciences et des techniques s'est toujours traduit par la volonté d'organiser de façon rigoureuse les connaissances scientifiques, ainsi que par le besoin de refaçonner les langues naturelles afin que celles-ci répondent à cette nouvelle tendance prescriptive:

It has been noted that "scientific knowledge as it was then coming into being needed to be organised around systems of technical concepts arranged in strict hierarchies of kinds and parts" - quite unlike "commonsense knowledge which can tolerate - indeed depends on - compromises, contradictions and indeterminacies of all kinds". (Halliday et Martin 1993: 6, cité dans Antia 2007: XIII) 
Les scientifiques sont en effet confrontés, à de nombreuses reprises, à des vides lexicaux et conceptuels dans une science qui avance à grande vitesse, en même temps qu'elle innove et expérimente. Les concepts ou objets scientifiques sont produits en masse et les terminologies scientifiques donnent lieu à des représentations multiples et polymorphiques, selon les horizons culturels en jeu. L'appréhension des univers différant d'une culture à l'autre, les concepts associés aux termes sont mouvants lorsqu'il s'agit d'innovation. Un terme peut recouvrir plusieurs déclinaisons de sens selon les univers de représentation. Des chemins dénominatifs variés peuvent être empruntés (Freixa Aymerich, Silva Fernández, et al. 2008), même pour un objet identique, en particulier lorsqu'il s'agit de sciences innovantes.

Enfin, l'industrie connaît également des besoins terminologiques forts, y compris en dehors de la normalisation technique. Ainsi, la tendance, au sein des grands groupes, est de plus en plus à l'élaboration de ressources linguistiques dans une volonté d'harmonisation des unités terminologiques et de leurs significations pour l'entreprise au niveau de sa représentation mondiale. Le sens des termes doit être appréhendé avec la même précision technique d'un lieu à l'autre. La terminologie a donc bel et bien une vie très active dans tous les univers humains: politiques, sociaux, culturels, économiques et scientifiques.

Mais, si dans le contexte des institutions gouvernementales et supra-gouvernementales, terminologie et traduction (et donc prise en compte des dimensions culturelles et interculturelles) sont intrinsèquement liées, notamment du fait du multilinguisme européen et international (citons les travaux de la Direction Générale de la Traduction de la Commission européenne ou encore les services de traduction de l'ONU), il n'en va pas de même dans les mondes scientifique et entrepreneurial. Ainsi, la très grande majorité des publications scientifiques sont rédigées en anglais, langue véhiculaire par essence, ce qui induit un réel besoin terminologique non satisfait, en l'occurrence, par le biais de la traduction. Les bases de données terminologiques (BDT) jouent donc un rôle essentiel en tant qu'instrument de facilitation des échanges dans la société de la connaissance, de l'économie, de la science et de la politique.

Par ailleurs, les besoins en matière de précisions sémantiques se font de plus en plus pressants. À l'heure de la mondialisation, de la multiplication des activités dans tous les domaines et de la rapidité de développement des concepts sur l'ensemble de la planète, les acteurs de la société de la connaissance, de l'économie et de la politique ont de plus en plus besoin de clarifications, de précisions, de définitions, de mise en relation des concepts et des idées et connaissances qu'ils véhiculent. Or, les solutions apportées par la terminologie traditionnelle ne semblent plus satisfaire complètement les principaux intéressés. Sur le seul plan opérationnel, les délais d'édition des bases de données terminologiques et des dictionnaires sont très longs. La concomitance entre évolution sociétale et progression scientifique devient donc difficile. Qui plus est, lorsque les terminologies sont posées, le plus souvent les usages viennent malmener le travail des terminologues, usages qui se fondent sur le réel et tentent, par une sorte d'improvisation de première nécessité, de répondre à des besoins, de combler des manques, de fournir du langage et du sens à des univers qui, sans cela, ne pourraient avancer.

En outre, les difficultés viennent également de la masse actuelle des données à gérer et des coûts que cela engendre. En effet, partout dans le monde, des idées fusent et se croisent de sorte que la masse d'informations et la vitesse de transformation des 
données dans les différents domaines sont considérables. La société de la connaissance s'est métamorphosée tandis que la société de l'information a donné lieu à de nouveaux défis pour la terminologie. Les besoins ont changé et se sont diversifiés. Nombre d'entreprises recherchent désormais une uniformisation de leur terminologie que ce soit en production ou en stratégie. L'ingénierie linguistique et plus particulièrement la construction de ressources ontoterminologiques est devenue un véritable enjeu, doublé d'une activité incontournable pour les entreprises dont les besoins en matière de gestion et d'appréhension documentaires ne cessent de croître:

[...] la demande des entreprises s'est faite pressante. En effet, elles se sont retrouvées avec des volumes de données textuelles considérables à traiter, c'est-à-dire à rédiger, archiver, mettre à jour et surtout pour lesquelles il fallait vérifier la cohérence. Si on considère que la documentation concernant un satellite correspond à environ 250000 pages, on comprend qu'aucun humain ne peut vérifier la cohérence de ces documents. (Condamines 2008: 42)

En d'autres termes, la complexité et la multiplicité du savoir, associées aux problématiques d'inter- et de transdisciplinarité qui sont à présent les nôtres montrent clairement que la terminologie a cédé le pas aux terminologies, qui requièrent des méthodologies et des outils différents selon les utilisateurs et les usages de la communication moderne ainsi qu'une actualisation permanente du savoir.

\subsection{Terminologies et nouvelles technologies}

Il semble donc désormais nécessaire de poser en principe le fait que les besoins étant croissants, les réponses ne pourront être que diversifiées et modélisées différemment selon les domaines, les usages et les utilisateurs. Il est dorénavant nécessaire, avant de se lancer dans un projet de terminologie, d'analyser non seulement l'objet ou le domaine lui-même, mais l'utilisation et les développements liés aux besoins des utilisateurs et à leurs modes de communication. La recherche dans ce domaine aujourd'hui est donc de plus en plus empirique et tournée vers l'innovation, même si les théories structurelles du domaine constituent les fondamentaux méthodologiques. La convergence de la linguistique, de l'intelligence artificielle (IA), de l'ingénierie des connaissances et enfin des sciences de l'information ont en effet permis de remettre en question la manière dont la modélisation des connaissances était envisagée jusqu’à présent en terminologie. Sur un plan dictionnairique, le débat terminologique a longtemps porté sur le choix des contenus, à savoir sur la nature des informations devant figurer dans un dictionnaire technique, avec une opposition latente entre données lexico-sémantiques et encyclopédiques (Mel'čuk 1988; McArthur 1986). Mais, avec l'émergence de l'informatique et notamment du format textuel électronique, ce débat a laissé la place à la question de la structuration des données terminographiques. Il est d'ailleurs intéressant de noter, à titre indicatif, que ce rapprochement interdisciplinaire était déjà annoncé à l'époque par les partisans de la TGT:

Étant donné que les études sur l'intelligence artificielle et la mise au point de systèmes experts sont amenées à traiter des systèmes de notions, des combinaisons de notions, de la représentation conceptuelle de la réalité, etc., il faut s'attendre à un renforcement des affinités entre la théorie générale de la terminologie et l'informatique dans un avenir proche. (Felber 1987: 91, cité dans Condamines 2003: 46) 
Toujours est-il que le développement de nouveaux supports et applications documentaires tels que les thésaurus pour l'indexation automatique, les réseaux de termes et constructions d'index utilisés dans les moteurs de recherches, les index hypertextuels, les bases de données lexicales type Wordnet ou encore les ontologies (Aussenac-Gilles 2004; Aussenac-Gilles et Condamines 2004), pour les plus modernes d'entre eux, ont mis en exergue les insuffisances structurelles des BDT classiques et la nécessité d'élaborer un nouveau modèle de données terminologiques.

L'une des principales faiblesses dénoncées par nombre de linguistes (Meyer et Skuce 1990; Otman 1996; Condamines 2003) est l'incapacité des bases traditionnelles à établir et à fournir une représentation explicite de la structure conceptuelle des domaines qu'elles sont censées décrire; une absence d'autant plus surprenante, étant donné la place prépondérante qu’occupe le concept en terminologie (Lerat 1988: 12). En effet, l'analyse conceptuelle a toujours constitué une part essentielle du travail terminologique, car elle permet d'appréhender et de structurer des connaissances diffuses et fragmentées:

Knowledge does not come "off the shelf, prepackaged, ready for use" (Hayes-Roth 1987: 293). As already mentioned, it can be inconsistent and contradictory. It can be multidimensional, since experts' understanding of a conceptual system can depend on their point of view. It may be hard to "capture", since it is constantly changing, and since emergent knowledge can be incomplete and unclear. (Meyer, Skuce, et al. 1992: 957)

Comprendre et faire comprendre des concepts spécialisés implique d'appréhender les interrelations entre ces derniers afin d'orienter correctement la recherche de documents auprès des experts, de communiquer efficacement avec ceux-ci, d'être en mesure d'identifier les phénomènes de synonymie, d'élaborer des définitions, d'établir des équivalents, de gérer la néologie, de réviser ou encore de mettre à jour les fiches terminologiques. Or la structure des bases de données traditionnelles est dépourvue de systèmes de représentation relationnelle des concepts. En effet, l'information conceptuelle y est disponible seulement de manière implicite, sous la forme de définitions, de contextes ou encore d'étiquettes, c'est-à-dire en langage naturel et donc inexploitable de manière automatique. Qui plus est, la majorité des données fournies sont d'ordre linguistique, restreignant de fait ses utilisateurs potentiels à une catégorie unique d'usagers, à savoir les traducteurs (Meyer, Skuce, et al. 1992: 956).

Par ailleurs, les définitions, les relations conceptuelles, les arbres de connaissance et l'ensemble des informations linguistiques sont toujours rattachés à des problématiques ou à des spécificités interculturelles. Quelle que soit la forme de l'objet de recherche, quel que soit le domaine, les concepts et les termes associés (simples ou en chaînes sémantiques) sont dépendants des univers de pensée et de croyance (DikiKidiri 2002). Ce sont ces univers et leur explication et mise en perspective qu'il est essentiel d'intégrer dans les nouvelles bases de connaissances multilingues spécialisées. Or, contrairement à ce que l'on pourrait penser, un dictionnaire classique même électronique ou une base de données un tant soit peu élaborée ne permettent pas de rendre compte de cette dimension interculturelle, à l'instar de la base IATE:

Il s'agit bien évidemment d'une initiative terminologique indispensable et tout à fait louable, mais qui trouve néanmoins ses limites dans le contenu, la nature et la structure de la base proposée. En effet, IATE est une base de données terminologique dite classique: elle se caractérise par un nombre de champs terminologiques extrêmement limité: une marque de domaine, une définition, un équivalent unique et, à certaines 
reprises, un contexte, lorsque ce dernier est disponible. Elle est totalement dépourvue de données d'ordres conceptuel et interculturel, qui sont, nous l'avons vu, indispensables pour saisir les subtilités de la langue et de la terminologie juridique. (Peraldi 2011 : 35)

Ces dictionnaires font état, dans le meilleur des cas, de certaines similitudes ou connotations culturelles et interculturelles liant les équivalents d'un même concept.

\section{Approches méthodologiques}

\subsection{Une approche textuelle}

La définition des contenus terminologiques et de leur structuration impose également de s'interroger sur le cadre théorique sous-jacent à toute méthodologie aussi empirique soit-elle. Nous savons aujourd'hui que l'approche entièrement conceptuelle de la TGT n'a pas résisté aux nouvelles pratiques terminographiques, liées notamment à l'émergence de la linguistique de corpus basée sur une observation des faits de langue, ni aux nouveaux courants de pensée qui ont investi le champ de recherche de la terminologie. La socioterminologie, la terminologie cognitive et la terminologie textuelle notamment ont montré que l'approche structurelle de l'école de Vienne était trop restrictive et orientée sur les aspects formels de la langue, pour pouvoir rendre compte de la spécificité des termes (Cabré 1998), réhabilitant par la même occasion l'analyse discursive, la phraséologie ou encore une analyse lexico-syntaxique des termes et se traduisant, de fait, par un rapprochement incontestable entre terminologie et linguistique. Il semblerait que le débat terminologique se soit déplacé, aujourd'hui vers la question du «tout textuel» ou du «tout ontologique». La convergence de la linguistique et de l'intelligence artificielle a non seulement permis de remettre en cause la modélisation des bases de données, au bénéfice de ce que nous appelons les bases de connaissances terminologiques (BCT), mais elle a également permis d'asseoir l'importance de l'approche textuelle dans la terminologie. En effet, au lieu d'aller des «objets vers leur nomination» (Aussenac-Gilles et Condamines 2007: 4), cette nouvelle démarche part des discours réels vers les ressources terminologiques ou ontologiques ainsi créées, en vue d'une meilleure utilisation de ces dernières et d'un accès accru et automatique aux informations qu'elles recèlent, justifiée d'ailleurs par la masse des informations à traiter et à décrire telle que nous l'évoquions plus tôt:

Depuis une quinzaine d'années, les textes sont utilisés comme vecteurs de connaissances pour construire des terminologies ou des ontologies. L'utilisation de textes est venue se substituer à l'interrogation d'experts ce qui a permis de mettre en œuvre des méthodes d'extraction automatique de connaissances. La thématique de la construction de ressources terminologiques, soutenue par une demande sociétale de la gestion de la documentation très prégnante, s'est ainsi fortement développée. (Condamines et Jacques 2006: 185)

Pourtant, cet engouement textuel au sein de la représentation des connaissances n'est pas partagé par tous. En effet, d'après Roche (2007: 162), l'élaboration d'une terminologie et plus particulièrement d'un réseau conceptuel à partir d'un corpus, c'est-à-dire un ensemble de textes partiel et incomplet, constitue la principale faiblesse de toute démarche protextuelle, car elle fait de l'ontologie une entité/ressource difficilement partageable et reproduisible. Selon lui, les termes décrits dans les corpus ne 
reflètent pas des concepts, mais uniquement des usages linguistiques propres à certaines communautés discursives. Dans la lignée de Rastier (2004), le linguiste estime qu'il est impossible de projeter une structure conceptuelle sur une structure lexicale, autrement dit le lexique ne peut en aucun cas traduire une représentation scientifique du monde:

On n'insistera jamais assez sur le fait qu'il ne faut pas confondre la conceptualisation d'un domaine avec les discours auxquels elle peut donner lieu. Les connaissances scientifiques sont nécessaires à la compréhension des textes de spécialité, elles ne peuvent donc en être extraites: "Car on ne voit jamais personne devenir médecin par la simple étude des recueils d'ordonnances» (Aristote, Ethique à Nicomaque, X, 10, 1181b). (Roche 2008: 55)

En d'autres termes, la compréhension des discours scientifiques requiert des connaissances extralinguistiques ne figurant pas dans les textes (métaphores, figures de rhétorique, etc.) qui s'avèrent problématiques lorsqu'il s'agit d'en extraire des informations et de les conceptualiser, car elles supposent que l'auteur et le lecteur partagent une conceptualisation et une compréhension commune du monde. S’impose donc la nécessité de définir une structure notionnelle unique pour les différentes communautés de pratique partageant la même réalité (une ontoterminologie); en d'autres termes, une conceptualisation dans un langage formel, émanant directement des experts et permettant la création d'une ontologie davantage universelle et partageable puisque non dépendante des manifestations lexicales d'une communauté discursive. En réalité, Roche prône ici une réhabilitation complète de la notion de concept et notamment le retour à un système de représentation des connaissances tel qu'il était envisagé par Wüster et ses adeptes.

Les partisans de l'approche textuelle, à l'instar d'Aussenac-Gilles et Condamines (2009), admettent également l'instabilité latente des discours ainsi que la part d'implicite et donc d'interprétation qu'engendre la structuration d'une terminologie élaborée à partir de données textuelles:

Les discours en effet ne sont pas stables, contrôlables et définissables comme le sont les objets référentiels et travailler à partir de textes, c'est être confronté à la variation et à la nécessité d'interpréter: "Au lieu de partir d'une ontologie préfixée, dont le texte ne serait qu'une manifestation toujours partielle et imparfaite [la conception rhétorico/ herméneutique] cherche à faire émerger corrélativement des régularités et des singularités, et à leur faire correspondre, par construction interprétative, des fonds et des formes sémantiques.» (Aussenac-Gilles et Condamines 2009: 118, citant Rastier 2001 : 90)

Nous ne prétendons pas non plus à l'unicité de cette approche ontotextuelle. Mais la mise en œuvre de méthodes d'extraction automatique, associée à la puissance de l'outil informatique, justifie pleinement et à juste titre son recours. En effet, l'utilisation des textes comme vecteurs de connaissances et source de représentation conceptuelle favorise largement une exploitation rapide et efficace des données conceptuelles et langagières contenues dans les textes, répondant ainsi aux exigences croissantes des entreprises, ainsi qu'illustrées plus tôt. D’autre part, malgré la variation et les approximations liées à un corpus, le texte demeure le lieu d'expression privilégié des spécialistes d'un domaine spécifique et c'est notamment à travers les termes, mais aussi à travers leur environnement immédiat, autrement dit la phraséologie, qu'il est possible de déduire toute l'articulation de la pensée propre à une communauté 
discursive spécifique, notamment par la prise en compte d'éléments extralinguistiques tels que le genre textuel.

Tout en prônant la nécessité d'une prise de distance par rapport aux textes et aux éléments lexicaux servant à l'élaboration de toute structure conceptuelle, le recours à une exploration textuelle outillée (méthodes ascendantes et descendantes) nous semble constituer l'approche méthodologique la plus adéquate et la plus proactive dans la mesure où seule l'analyse contextuelle des textes spécialisés permet de conduire à des informations d'ordre linguistique et sémantique riches et multiples.

\subsection{Une approche pluridisciplinaire et collaborative}

Afin de rendre l'ensemble de ces informations accessible de façon aisée et ergonomique par les utilisateurs, il s'avère essentiel, par ailleurs, de passer par la technologie et par un nouveau système de gestion des connaissances. En effet, la profusion de connaissances qui pourront être mises en relation par des associations, dissociations, affinements des variantes et des spécificités, par zone géographique, domaine de spécialité, genre, etc., risque à terme d'être inutilisable et donc inutilisée. C'est la raison pour laquelle nos chercheurs tentent de travailler en parallèle et en collaboration avec des centres de recherche en systèmes d'information sur les meilleurs outils susceptibles de permettre une utilisation efficace des données spécialisées et de l'ensemble des liens établis à tous les niveaux entre elles, et ce, en fonction des métiers où ces données sont susceptibles d'être utilisées.

Il s'agit de créer des outils au service des utilisateurs et de leurs spécificités et d'appuyer la création de ces outils sur des travaux d'expertise reposant sur des échantillons d'utilisateurs et des audits concernant leurs besoins. Nous employons également le terme terminologies car à chaque projet sa spécificité: outils au service des traducteurs dans le cadre des mémoires de traduction ou des outils phraséologiques; bases de données utilisées par les industriels pour la rédaction technique dans le cadre d'outils spécifiques développés par des entreprises, utilisation des chaînes sémantiques (terminologiques et phraséologiques) au service des développements web : référencement, chaînes de connaissances, gestion des données du Web 2.0 et autres développements pour les domaines de spécialités, bases de connaissances pour les sciences, le droit, la traduction et l'interprétation, etc. Il s'avère indispensable de mener des études sur les besoins, de constituer un cahier des charges très précis et de s'interroger sur la mise en œuvre de l'expertise terminologique intégrée à des outils pour répondre auxdits besoins. Les terminologies sont donc inséparables des technologies associées, qu'il s'agisse du recours à des logiciels spécifiques, de développements de la gestion des données sur le web ou d'applications de la téléphonie mobile, etc.

La recherche appliquée sur les terminologies telle qu'elle est pratiquée au sein du CRATIL repose sur cette conception collaborative et plurielle. L'état de l'art, les modélisations, le transfert d'innovation, les partenariats de recherche sont au service de cette nouvelle dimension de la terminologie. Ainsi, du fait de la diversification des activités et des relations incontournables aujourd'hui entre terme, sens, univers de connaissances et univers de pensée, les terminologies font désormais partie intégrante de l'un des axes de recherche de la traductologie. Elles sont une branche à part entière, mais aussi un domaine de spécialité de la traductologie tout comme les mathématiques font partie intégrante de l'ingénierie. Les projets de recherche dans 
cette nouvelle perspective sont donc systématiquement pluridisciplinaires, associant les compétences des chercheurs du CRATIL aux compétences développées dans les centres de recherche partenaires : à savoir, deux des domaines de spécialité que nous étudions (droit, chimie, nanotechnologies, etc.) et les centres de recherche en systèmes d'information, et ce, afin de produire des outils opérationnels dont toute la valeur ajoutée repose sur une association des contenus (orientés utilisateurs) et des contenants (également orientés utilisateurs).

\subsection{Exemplification}

Les projets de recherche issus de ces associations ont maintenant trois ans et offrent des développements innovants. Les terminologies associées aux nouvelles technologies sont en effet l'innovation de l'ingénierie de demain au service de la gestion multilingue et interculturelle des connaissances.

Dans la lignée des critiques émises à l'encontre des bases de données classiques, notre choix s'est donc porté sur l'implémentation de bases de connaissances terminologiques. Pour rappel, les bases de connaissances, telles qu'elles sont pensées en IA, ont pour objectif de modéliser et de stocker de manière informatique un ensemble de connaissances, idées, concepts ou données et de permettre leur consultation/ utilisation. Elles servent à rassembler, de manière centralisée, l'expertise d'un domaine généralement formalisée de manière déclarative par le biais de règles, de faits ou de représentations, ce qui constitue un premier point de contact évident avec la terminologie. D’après Condamines (2003: 50), cinq éléments spécifiques caractérisent les BCT et constituent, à nos yeux, autant de marqueurs d'évolution des bases de données classiques: l'approche textuelle, la mise en réseau des termes, la distinction terme/concept, la notion d'usage mentionnée précédemment et enfin le caractère formalisateur des bases de connaissances.

En effet, la richesse et la diversité des relations conceptuelles (c'est-à-dire au-delà du lien classique «est une sorte de») figurant dans une base représentent des critères de modernité en matière de modélisation des connaissances. La distinction terme/concept permet non seulement la mise en relation des niveaux lexical et conceptuel au sein de la BCT, réglant de fait la question des contenus terminologiques, mais elle permet également de gérer les phénomènes de polysémie, synonymie et homonymie jusqu'alors ignorés dans le traitement terminologique des domaines de spécialité. Quant à la notion d'usage, elle permet de placer l'utilisateur au cœur du processus d'élaboration de l'outil, en contraignant ses concepteurs à s'interroger sur la méthodologie d'approche de l'application ainsi créée et sur ses utilisations potentielles. Enfin, l'obligation de formalisation des bases de connaissances oblige à décontextualiser les informations linguistiques, en permettant notamment de s'affranchir de la dimension introspective qui caractérisait les bases de données classiques, souvent élaborées via l'interrogation et la validation d'un seul et même panel d'experts.

Plus spécifiquement, la méthodologie de recherche appliquée à l'élaboration des bases de connaissances multilingues combine trois savoir-faire. En premier lieu, elle a comme source de contenus et de validation des données, la recherche en traductologie et sa branche terminologique, qui est orientée sur l'usage de données multiples, multi-formats et multilingues et sur les mises en relation sémantiques, culturelles, 
ergonomiques, etc. Sur un plan méthodologique, les analyses linguistiques menant à la constitution de ces terminologies sont systématiquement fondées sur une exploration outillée sur corpus, impliquant notamment l'utilisation de marqueurs linguistiques (Pearson 1998; Candel 2000; L'Homme 2004), une décomposition sémantique comparative des énoncés définitoires (Thoiron, Arnaud, et al. 1996; Temmerman 2000) lorsque nous sommes confrontés à des phénomènes d'indétermination terminologique, de multidimensionnalité ou de variation, ainsi qu'une analyse lexicosémantique et paradigmatique des termes (L'Homme 2000a, 2000b) afin d'identifier les relations notionnelles à l'origine du réseau conceptuel à bâtir. Les arbres de concepts permettent en effet de représenter et d'organiser de manière graphique l'univers d'un concept (Wright 1997) afin de procurer une image plus «parlante» pour l'esprit quand le langage écrit et parlé atteint ses limites descriptives, mais aussi de mettre en exergue les incohérences ou difficultés notionnelles propres à chaque domaine de spécialité.

D'un point de vue technique, les travaux sont menés en partenariat direct avec la recherche en architectures informatiques et en particulier le SOA: Architecture orientée service. Une architecture orientée services (notée SOA pour Services Oriented Architecture) est une architecture logicielle s'appuyant sur un ensemble de services simples. L'objectif d'une architecture orientée services est de décomposer une fonctionnalité en un ensemble de fonctions basiques, appelées «services", fournies par des composants, et de décrire finement le schéma d'interaction entre ces services. Les travaux de recherche du CRATIL visent à créer des bases de connaissances au service des métiers dont les spécificités seront intégrées à la construction même de la base. Cela revient en quelque sorte à dessiner, tel un architecte, « une maison» (ou "un bâtiment») la plus ergonomique possible, dans laquelle l'accès aux données (quelles qu'elles soient) se fera de la manière la plus opérationnelle pour les utilisateurs métiers. Afin de formaliser ces travaux en un objet réel et exploitable, le centre de recherche s'associe à des centres de recherche en systèmes d'information qui eux travaillent simultanément sur la réalisation technique du produit fini (pour ainsi dire la "construction» de la maison). Le succès de cette méthodologie pluridisciplinaire est qu'elle est collaborative et associative, les avancées des uns et des autres s'enrichissant des apports des uns et des autres.

Actuellement, parmi l'ensemble des projets de recherche menés ces dernières années, trois bases de connaissances spécifiques sont en cours d'élaboration: l'une portant sur les nanotechnologies et les nanosciences en collaboration avec le CNRS et le centre de recherche de l'ISEP (Institut d'électronique de Paris) (Peraldi 2010 ${ }^{8}$; une autre, centrée sur le domaine des énergies renouvelables, en collaboration avec un des centres de recherche de l'INSA de Rouen, le LITIS (Laboratoire d'Informatique, du Traitement de l'Information et des Systèmes), l'unité de recherche dans le domaine des sciences et technologies de l'information de Haute-Normandie.

Enfin, le dernier projet (Peraldi $2008^{\circ}$ ) a été mis en place à l'initiative de l'association Juriconnexion, une association ouverte à toutes personnes (juristes, documentalistes, avocats, notaires, administrations, banques, grandes entreprises, collectivités territoriales) utilisant divers produits électroniques d'information juridique dans le cadre de leur pratique professionnelle quotidienne. Depuis 1988, Juriconnexion entretient un dialogue constructif avec les producteurs, serveurs et distributeurs. Elle exprime le point de vue des utilisateurs auprès des pouvoirs publics 
et joue un rôle actif dans la construction des banques de données juridiques officielles. Elle travaille en liaison avec d'autres associations ou organismes ayant des préoccupations voisines. La méthodologie de recherche a été élaborée, un premier réseau sémantique bilingue a été construit dans les sous-domaines du droit des contrats électroniques, une réflexion sur les utilisateurs a été lancée. Le projet est actuellement en attente de financement pour un projet européen collaboratif.

Les contenus juridiques accessibles sont nombreux: références aux différentes lois, réglementations et jurisprudences pour un même référent juridique dans différentes cultures et commentaires interculturels pouvant permettre une meilleure intercompréhension des données et favoriser leur exploitation.

Le point de départ de l'ensemble de ces travaux est l'analyse des besoins en matière de gestion des connaissances et plus particulièrement des connaissances multilingues dans un domaine de spécialité. Les méthodes de cette recherche empirique permettent, sur le terrain, d'effectuer des audits autour des métiers ciblés, puis d'analyser les besoins par activités et sous-domaines. Il s'agit de décrire des pratiques et des besoins à partir d'échantillons d'utilisateurs de services différents d'un même métier. La recherche appliquée entre alors en jeu avec la construction par les terminologues des arborescences bilingues ou multilingues qui permettront d'élaborer les contenus, conformément à l'analyse des besoins. Cette méthodologie, née de la confluence des travaux de terminologie, de sémantique et de linguistique, utilise depuis peu les apports de la traductologie pour l'interrelation entre les réseaux conceptuels interculturels. Cette représentation facilite l'apprentissage et l'appropriation de concepts fluctuants, en structurant et en liant un grand nombre d'informations multilingues. Nous insistons sur le fait que la dimension interculturelle conférée à nos ressources repose sur le savoir-faire spécifique des traducteurs/terminologues chercheurs. Par ailleurs, la constitution de ces ressources ontologiques s'appuie également sur la validation des chercheurs en ingénierie. En effet, bien que la collaboration avec ces derniers puisse sembler inopportune, c'est précisément ce travail collaboratif entre des spécialistes de la structuration des outils et les spécialistes de la structuration des contenus qui permet l'optimisation de la mise en forme des savoirs en vue d'une utilisation qui sera elle aussi optimisée. Il est aujourd'hui devenu d'actualité de parler de pluridisciplinarité: c'est en effet dans cette action de mise en commun des savoir-faire dans le cours même de la recherche que réside l'innovation. En d'autres termes, toute analyse linguistique, qui plus est lorsqu'il s'agit de discours spécialisés, doit systématiquement être pensée comme une co-construction interprétative qui a lieu entre le terminologue et l'expert (Condamines 2003). Le linguiste se sert des indices linguistiques à sa disposition pour construire et proposer une théorie autour des phénomènes langagiers relevés tandis que l'expert joue un rôle de partenaire (Picton 2009), en validant et éventuellement en complétant les structures préétablies.

Une fois les cartes conceptuelles élaborées, les architectures métiers dessinées et la modélisation informatique développée, la base peut voir le jour. Les technologies employées pour la réalisation sont nombreuses, mais il est également possible de travailler la création de façon adaptée, non seulement en fonction des utilisateurs métiers (scientifiques, chercheurs, juristes, traducteurs), mais également en fonction des utilisations géographiques et régionales (différentes ergonomies selon des lieux d'utilisation). 


\section{Conclusion : développements possibles}

L’ensemble des ressources présentées sont multilingues et peuvent donner lieu à de nombreux développements. En effet, l'avenir de ces bases de connaissances repose sur leur utilisation et leur maintenance, induisant des mises à jour régulières des données et systèmes. Une des difficultés de notre société de la connaissance est, nous l'avons dit, la rapidité de l'évolution des données et des concepts, les innovations permanentes et les mouvances des terminologies et des acceptions. Dans un tel mouvement, les bases de connaissances multilingues et multimédias que nous élaborons devront être non pas statiques, mais évolutives. Pour ce faire, des développements sont à l'étude au niveau des utilisations de wikis et du Web 2.0. Par ailleurs, ces bases de connaissances pourront intégrer des outils divers: espaces multimédias pour des visualisations de cartes sémantiques, des images ou des documents audiovisuels; espaces pour des outils spécifiques: logiciel de rédaction technique, de localisation, de TAO par exemple. Les pistes de développement sont nombreuses. Il s'avère que le besoin est réel, les moyens existent et, qui plus est, différents univers professionnels requièrent des outils performants pour la gestion des connaissances multilingues. Ces outils favoriseront l'organisation des connaissances et l'exploitation des savoirfaire dans des domaines spécifiques répartis sur différentes zones culturelles. Ils seront également d'une grande aide pour les traducteurs et interprètes et pour les métiers qui seront concernés.

La traductologie et sa branche «terminologies» est depuis toujours au service du développement et de l'innovation dans l'ensemble des domaines requérant une organisation et une modélisation de l'usage du sens à travers des mots, des phrases, des idées, des concepts, des façons de concevoir et de dire le monde. Le monde a changé, les métiers de la traduction aussi, mais ils sont plus que jamais au cœur des développements de la société de la connaissance. C'est pourquoi la recherche au sein du CRATIL a pour stratégie l'innovation et propose une interdisciplinarité scientifique aussi bien au niveau des savoirs et des savoir-faire qu'au niveau méthodologique, en mettant en commun les techniques de la recherche empirique, de la recherche appliquée et de la recherche fondamentale.

\section{NOTES}

1. Die terminologische Gemeinschaftsarbeit darf sich nicht darauf beschränken, festzustellen, was ist. Sie muss danach trachten, den so widerspruchsvollen Sprachgebrauch zu vereinheitlichen und zu verbessern. (Wüster 1979/1985: 89)

2. IATE (Interactive Terminology for Europe). Consultée le 27 février 2012, <http://iate.europa.eu/ iatediff/switchLang.do ?success=mainPage\&lang $=\mathrm{fr}>$.

3. EuroVoc (Thésaurus multilingue de l'Union européenne). Consultée le 27 février 2012, < http:// eurovoc.europa.eu/drupal/ ?q=fr/node $>$.

4. UNTERM (United Nations Multilingual Terminology Database). Consultée le 27 février 2012, $<$ http://unterm.un.org/>.

5. L'aménagement linguistique peut être défini comme une intervention humaine volontaire sur la langue, destinée à infléchir son usage à des fins de politique linguistique et plus spécifiquement de protection de la langue nationale (Auger 1984; Depecker 1996).

6. La normalisation linguistique est de fait souvent perçue comme un processus extrêmement pesant et est comparée à du dirigisme linguistique (Rondeau 1984: 115; Depecker 1996); alors que la terminologie (du moins telle qu'elle est envisagée aujourd'hui) est dite descriptive, autrement dit le terminologue observe et analyse l'émergence des termes, la normalisation est prescriptive et réflexive. 
7. UNESCO (2005): Principes directeurs sur les politiques en matière de terminologie. Élaboration et mise en œuvre des politiques en matière de terminologie dans les communautés linguistiques. Paris: Organisation des Nations Unies pour l'éducation, la science et la culture. Consultée le 27 février 2012, <http://unesdoc.unesco.org/images/0014/001407/140765f.pdf>.

8. Peraldi, Sandrine (2010): De la traduction des nanosciences. (Journées d'études Traduire la diversité: domaines littéraire, juridique et des sciences de la vie, Université de Liège, 6-8 mai 2010). Communication non publiée.

9. Peraldi, Sandrine (2008): Ontologie juridique multilingue. (Table ronde aux Journées Européennes d'Informatique Juridique Mieux légiférer et mieux accéder au droit dans l'espace juridique européen grâce aux évolutions technologiques, Paris, 10-12 décembre 2008). Communication non publiée.

\section{RÉFÉRENCES}

Antia, Bassey Edem (2007): Indeterminacy in Terminology and LSP: Studies in Honour of Heribert Picht. Amsterdam/Philadelphia: John Benjamins.

Auger, Pierre (1984): La Commission de terminologie de l'Office de la langue française et la normalisation terminologique. Terminogramme. 26(7):9-12.

AussenaC-Gilles, Nathalie (1999): Gediterm: un logiciel pour gérer des bases de connaissances terminologiques. Terminologies nouvelles. 19:111-123.

Aussenac-Gilles, Nathalie (2004): Représentation sémantisée des textes: terminologies et dimensions pragmatiques (qui-quand-où). In: Marie-Hélène Antoni-Lay et François Yvon, dir. Actes du Colloque International sur la Fouille de Textes. (CIFT 2004, La Rochelle, 23-25 juin 2004). Paris: ENST, 33-39.

Aussenac-Gilles, Nathalie et Condamines, Anne (2001): Entre textes et ontologies formelles: les bases de connaissances terminologiques. In: Manuel Zacklad et Michel Grundstein, dir. Ingénierie et capitalisation des connaissances. Paris: Hermes, 153-176.

Aussenac-Gilles, Nathalie et Condamines, Anne (2004) : Documents électroniques et constitution de ressources terminologiques ou ontologiques. Information, Interaction, Intelligence. 4(1):75-92.

Aussenac-Gilles, Nathalie et Condamines, Anne (2007): Corpus et terminologie. In: Roger T. PÉDAuQue, dir. La redocumentarisation du monde. Toulouse: Cepadues Editions, 131-147.

Aussenac-Gilles, Nathalie et Condamines, Anne (2009): Variation syntaxique et contextuelle dans la mise au point de patrons de relations sémantiques. In: Jean-Luc MineL, dir. Filtrage sémantique. Paris: Hermes/Lavoisier, 115-149.

CABré, Maria Teresa (1998): La terminologie, théorie, méthodes et applications. Ottawa: Presses de l'Université d'Ottawa.

Candel, Danielle (2000): La définition chez les scientifiques. Terminologies Nouvelles. 21:52-57.

Candel, Danielle (2004): Wüster par lui-même. In: Colette Cortès, dir. Des fondements théoriques de la terminologie. Cahiers du C.I.E.L. (7):15-32.

Condamines, Anne (2003): Sémantique et corpus spécialisés: constitution de bases de connaissances terminologiques. Mémoire d'Habilitation à diriger des recherches. Toulouse: Université de Toulouse 2.

Condamines, Anne (2008) : Peut-on prévenir le risque langagier dans la communication écrite? Langage et Société. 125:39-52.

Condamines, Anne et Jacques, Marie-Paule (2006): Le repérage de l'hyperonymie par un faisceau d'indices: mise en question de la notion de "marqueur». In: Rémi LeHN, Mounira Harzallah, Nathalie AussenaC-Gilles, et al., dir. Actes de la SdC 2006. (Journée Textes et Connaissances, Semaine de la connaissance, Nantes, 26-30 juin 2006). Maison d'édition non spécifiée, 185-194.

Depecker, Loïc (1996): Terminologie et standardisation. Hyeronymus Complutensis. 3:85-88.

Diki-Kidiri, Marcel (2002): La terminologie culturelle, fondement d'une localisation véritable. Actas del VIII Simposio Iberoamericano de Terminología. (Carthagène des Indes, 28-31 octobre 2002). Maison d'édition non spécifiée, cédérom.

Felber, Helmut (1987): Manuel de terminologie. Paris: UNESCO. 
Freixa Aymerich, Judit, Fernández Silva, Sabela et Cabré Castellví, Maria Teresa (2008): La multiplicité des chemins dénominatifs. Meta. 53(4):731-747.

Gambier, Yves (1991): Présupposés de la terminologie: vers une remise en cause. Cahiers de linguistique sociale. 18:31-58.

Gaudin, François (1993): Pour une socioterminologie. Des problèmes sémantiques aux pratiques institutionnelles. Rouen: Université de Rouen.

GAUDIN, François (2003): Socioterminologie: une approche sociolinguistique de la terminologie. Bruxelles: De Boeck/Larcier/Duculot.

Halliday, Michael Alexander Kirkwood et Martin, J. R., dir. (1993): Writing Science. Literacy and Discursive Power. Pittsburgh: University of Pittsburgh Press.

Hayes-Roth, Frederick (1987): Expert Systems. In: Stuart Shapiro, dir. Encyclopedia of Artificial Intelligence. New York: John Wiley and Sons.

Lerat, Pierre (1988): Terminologie et sémantique descriptive. La banque des mots. (Numéro spécial):11-30.

L'Hoмme, Marie-Claude (2000a): Understanding Specialized Lexical Combinations. Termino$\log y .6(1): 89-110$.

L'Homme, Marie-Claude (2000b): Les enseignements d'un mot polysémique sur les modèles de la terminologie. Cahiers de grammaire. 25:71-91.

L'Hoмme, Marie-Claude (2004): Terminologie: principes et techniques. Montréal: Presses de l'Université de Montréal.

McArthur, Tom (1986): Worlds of Reference: Lexicography, Learning and Language from the Clay Tablet to the Computer. Cambridge: Cambridge University Press.

MeL'čuk, Igor (1988): Semantic description of lexical units in an explanatory combinatorial dictionary: Basic principles and heuristic criteria. International Journal of Lexicography. 1(3):165-188.

Meyer, Ingrid, Skuce, Douglas, Bowker, Lynne, et al. (1992): Towards a new generation of terminological resources: An Experiment in building a terminological knowledge base. Proceedings of the $14^{\text {th }}$ International Conference on Computational Linguistics. (COLING 92, Nantes, 23-28 août 1992), Morristown: International Committee of Computational Linguistics, 956-957.

Otman, Gabriel (1996): Les représentations sémantiques en terminologie. Paris: Masson.

Pearson, Jennifer (1998): Terms in Context. Amsterdam/Philadelphia: John Benjamins.

Pecman, Mojca, Juilliard, Claudie, KüBler, Natalie, et al. (2010): Processing collocations in a terminological database based on a cross-disciplinary study of scientific texts. Proceedings of eLex2009. Cahiers du Cental. (22-24 octobre 2009) Louvain-la-Neuve: Université catholique de Louvain, 249-262.

Peraldi, Sandrine (2011). Traduire le droit: vers des normes de référencement européennes? In: Actes du colloque e-justice. Archives de philosophie du droit. 54:27-39.

Picton, Aurélie (2009): Diachronie en langue de spécialité. Définition d’une méthode linguistique outillée pour repérer l'évolution des connaissances en corpus. Un exemple appliqué au domaine spatial. Thèse de doctorat. Département Sciences du langage, Université Toulouse le Mirail - Toulouse II.

Rastier, François (2004): Ontologie(s). Revue d'Intelligence Artificielle. 18(1):15-40.

Roche, Christophe (2007): Dire n'est pas concevoir. In: Actes des $18^{e}$ journées francophones d'Ingénierie des Connaissances (IC2007, Grenoble, 4-6 juillet 2007). Toulouse: CépaduèsÉditions, 157-168.

Roche, Christophe (2008): Faut-il revisiter les principes terminologiques? In: Actes de la deuxième conférence TOTh (TOTh 2008, Annecy, 5-6 juin 2008). Annecy: Institut Porphyre, 53-72.

RondeAu, Guy (1984): Introduction à la terminologie. $2^{\mathrm{e}}$ éd. Chicoutimi: Gaëtan Morin Editeur. Skuce, Douglas et Meyer, Ingrid (1990): Concept Analysis and Terminology: A KnowledgeBased Approach to Documentation. In: Hans Karlgren. Proceedings of the Thirteenth International Conference on Computational Linguistics. (COLING 90, Université d'Helsinki, 20-25 août 1990). Vol. 1. Helsinki: Universitas Helsingiensis, 56-58. 
Szulman, Sylvie, BiéBow, Brigitte et Aussenac-Gilles, Nathalie (2001): Vers un environnement intégré pour la structuration de terminologies: TERMINAE. In: Actes des $4^{\mathrm{e}}$ rencontres internationales Terminologie et intelligence artificielle (TIA-2001, Nancy, 3-4 mai 2001), 98-108.

Temmerman, Rita (2000): Towards New Ways of Terminology Description - The Sociocognitive Approach. Amsterdam/Philadelphia: John Benjamins.

Thoiron, Philippe, Arnaud, Pierre, BÉJoInt, Henri, et al. (1996) : Notion d'«archi-concept» et dénomination. Meta. 41(4):512-524.

Wright, Sue Ellen (1997): Representation of Concept Systems. In: Sue Ellen Wright and Gerhard Budin, dir. Handbook of Terminology Management. Amsterdam/Philadelphia: John Benjamins, 89-97.

WÜSTER, Eugen (1979/1985): Einführung in die allgemeine Terminologielehre und terminologische Lexikographie. $2^{\mathrm{e}}$ éd. posthume. Copenhague: Copenhagen School of Economics. 